\title{
Proximity effect in normal metal-multiband superconductor hybrid structures
}

\author{
A. Brinkman and A. A. Golubov \\ MESA + Research Institute and Faculty of Science and Technology, University of Twente, P.O. Box 217, 7500 AE Enschede, \\ The Netherlands \\ M. Yu. Kupriyanov \\ Institute of Nuclear Physics, Moscow State University, 119992 Moscow, Russia \\ (Received 13 January 2004; revised manuscript received 25 February 2004; published 4 June 2004)
}

\begin{abstract}
A theory of the proximity effect in normal metal-multiband superconductor hybrid structures is formulated within the quasiclassical Green's function formalism. The quasiclassical boundary conditions for multiband hybrid structures are derived in the dirty limit. It is shown that the existence of multiple superconducting bands manifests itself as the occurrence of additional peaks in the density of states in the structure. The interplay between the proximity effect and the interband coupling influences the magnitudes of the gaps in a superconductor in a nontrivial way and can even give rise to an enhancement of multiband superconductivity by the proximity to a superconductor with a lower transition temperature. The developed theory is applied to the calculation of supercurrent in multiband superconductor-normal metal-superconductor Josephson junctions with low-transparent interfaces, and the results are compared with the predictions for multiband tunnel junctions.
\end{abstract}

DOI: 10.1103/PhysRevB.69.214407

PACS number(s): 74.20.-z, 74.45.+c, 74.70.Ad

The proximity effect is the phenomenon that a superconducting order parameter can penetrate from a superconductor $(S)$ into a normal metal $(N)$, or another superconductor $\left(S^{\prime}\right)$ with a critical temperature $T_{c S^{\prime}}<T_{c S}$, over a distance of the order of the coherence length, inducing a minigap in $N$ or $S$. This phenomenon is well understood, both in terms of Andreev reflections as well as in terms of microscopic Green's functions. $^{1-9}$

It is not known, however, how the proximity effect will manifest itself when multiple pairing potentials are present in the superconductor. This question has become relevant now that multiband superconductors are coming into practical use. The most clear example of a multiband superconductor is $\mathrm{MgB}_{2}$, for which the experimental and theoretical evidence for the coexistence of two gaps is overwhelming. ${ }^{10}$ The multiband nature of the superconductivity in $\mathrm{MgB}_{2}$ is theoretically well explained ${ }^{11}$ by the qualitative difference between different sheets of the Fermi surface, together with the large disparity of the electron-phonon interaction. Therefore, in this paper, the question is addressed how the multiband nature influences the proximity effect. For example, what will be the density of states in a $S N$ bilayer, where $S$ is a two-band superconductor?

Josephson and quasiparticle tunneling in hybrid structures containing multiband superconductors have been investigated theoretically in Ref. 12 and applied to the calculation of the total Josephson current in a SIS two-band Josephson tunnel junction. For all-MgB $\mathrm{Mg}_{2}$ devices, high-quality tunnel barriers are not available yet, and realizing $S N S$ structures is an attractive alternative, of which first systems have been realized already. ${ }^{13}$ In this paper, the theory of the multiband proximity effect is applied to the calculation of Josephson current in SNS structures having two-band $S$ electrodes. The practically interesting SINIS case is considered, where a nonideal interface transparency is taken into account. Predictions are made for Josephson devices based on $\mathrm{MgB}_{2}$ and com- pared with those for $\mathrm{MgB}_{2}$-based tunnel junctions.

In this paper, we will use the quasiclassical Green's function formalism in order to describe electrical transport in $S S^{\prime}$ hybrid structures, where $S^{\prime}$ is a single-band superconductor while $S$ is a multiband superconductor. We will restrict ourselves to the limit of diffusive transport, which is justified if $l_{S, S^{\prime}} \ll \xi_{S, S^{\prime}}$, where $l_{S, S^{\prime}}$ and $\xi_{S, S^{\prime}}$ are the electric mean free path and coherence length of the $S$ and $S^{\prime}$ materials respectively. In the dirty limit, the Green's functions in the $S^{\prime}$ metal are given by the standard Usadel equations. ${ }^{14}$ In the $S$ metal in the regime of vanishing interband scattering, as is the case for $\mathrm{MgB}_{2},{ }^{15}$ the Usadel equations take the form ${ }^{16}$

$$
\begin{gathered}
\frac{D_{S}^{\alpha}}{2 \omega G_{S}^{\alpha}}\left[\left(G_{S}^{\alpha}\right)^{2} \Phi_{S}^{\prime \alpha}\right]^{\prime}-\Phi_{S}^{\alpha}=-\Delta_{\alpha}, \\
\Delta_{\alpha}=2 \pi T \sum_{\beta, \omega \geqslant 0} \hat{\Lambda}_{\alpha \beta} \frac{G_{S}^{\beta} \Phi_{S}^{\beta}}{\omega} .
\end{gathered}
$$

Here, $\alpha$ and $\beta$ are the band indices, e.g., $\alpha, \beta=1,2$ in the two-band case (later we will use the band indices $\sigma$ and $\pi$ for $\mathrm{MgB}_{2}$ specifically), $\Delta_{\alpha}$ is the pair potential, $G_{S}^{\alpha}$ and $\Phi_{S}^{\alpha}$ are Green's functions, ${ }^{5} \omega=\pi T(2 n+1)$ are Matsubara frequencies, $D_{S}^{\alpha}$ is the diffusion coefficient, and $\hat{\Lambda}_{\alpha \beta}$ is the matrix of effective coupling constants. The prime denotes a derivative with respect to the coordinate $x$ in the direction perpendicular to the $S-S^{\prime}$ interface.

Equations (1) and (2) must in general be supplemented by boundary conditions. Zaitsev ${ }^{17}$ derived boundary conditions to the quasiclassical Eilenberger equations at the $S-S^{\prime}$ boundaries in the clean limit, which were further simplified in Ref. 5 in the dirty limit. These boundary conditions have to be modified when $S$ is a multiband superconductor. 
In the limit of small interband scattering a multiband superconductor may be represented by separate groups of superconducting electrons which interact with each other only indirectly, via self-consistent pair potentials in the bulk. Therefore, for the derivation of the boundary conditions for the Usadel equations, one can apply a similar procedure to that used in Ref. 5 in the single-band case. In the multiband case, the set of interface parameters $\gamma^{\alpha}$ and $\gamma_{B}^{\alpha}$, describing the proximity effect, should be introduced for each of the bands.

The first boundary condition relates the current from the $S^{\prime}$ metal side at the $S$ - $S^{\prime}$ interface, $\sigma G^{2} \Phi^{\prime}$, to that from the $S$ side, $\Sigma_{\alpha} \sigma_{S}^{\alpha}\left(G_{S}^{\alpha}\right)^{2}\left(\Phi_{S}^{\alpha}\right)^{\prime}$. Therefore we have

$$
\xi G^{2} \Phi^{\prime}=\sum_{\alpha} \frac{\xi_{S}^{\alpha}}{\gamma^{\alpha}}\left(G_{S}^{\alpha}\right)^{2}\left(\Phi_{S}^{\alpha}\right)^{\prime}
$$

with

$$
\gamma^{\alpha}=\frac{\rho_{S}^{\alpha} \xi_{S}^{\alpha}}{\rho \xi}, \quad\left(\xi_{S}^{\alpha}\right)^{2}=\frac{D_{S}^{\alpha}}{2 \pi T_{c S}}, \quad \xi^{2}=\frac{D}{2 \pi T_{c S}},
$$

in which from here on we drop all the $S^{\prime}$-indices for quantities in the $S^{\prime}$ layer. Here, $\sigma=1 / \rho$ and $\sigma_{S}^{\alpha}=1 / \rho_{S}^{\alpha}$ are the conductivities of the $S^{\prime}$ layer and the respective bands of the $S$ metal, $D$ is the diffusion constant in $S^{\prime}$ and $T_{c S}$ is the critical temperature of $S$. The ratio between the parameters $\gamma^{\alpha}$ for the different bands is mainly determined by the relation between the diffusion constants $D_{S}^{\alpha}$. In the case of $\mathrm{MgB}_{2}$, the $\pi$ band is generally considered to be more dirty than the $\sigma$ band, ${ }^{15}$ i.e., $D_{S}^{\pi} \ll D_{S}^{\sigma}$.

The second boundary condition relates the gradient of the Green's function $\Phi$ near the $S$ - $S^{\prime}$ interface to its jump at the interface due to the finite interface resistance. ${ }^{5}$ In the multiband case, this boundary condition yields, in accordance with current conservation, the equality of the current flowing in a single band superconductor $\sigma G^{2} \Phi^{\prime}$ and the sum of the currents injected into all bands of the multiband superconductor, thus giving

$$
\xi G \Phi^{\prime}=\sum_{\alpha} \frac{G_{S}^{\alpha}}{\gamma_{B}^{\alpha}}\left(\Phi_{S}^{\alpha}-\Phi\right),
$$

where $\gamma_{B}^{\alpha}=R_{B}^{\alpha} / \rho \xi$. $R_{B}^{\alpha}$ are the components of the specific interface resistance, describing the tunneling of an electron across the interface into the corresponding conduction band. This boundary condition is general and does not depend on the specific band structure of both materials.

In order to obtain the resistances $R_{B}^{\alpha}$, we have to evaluate the effective junction transparency components. It was first pointed out by Mazin, ${ }^{18}$ that the normal state conductance $R_{\alpha}^{-1}$, in the limit of a specular barrier with small transparency, is proportional to the Fermi-surface average $\left\langle N v^{2}\right\rangle_{\alpha}$, where $N$ is the density of states and $v$ the Fermi velocity. In Ref. 12 it was further shown that the normal state resistance component of tunneling into band $\alpha$ of $S$ is given by the contribution of the electrons in band $\alpha$ to the squared plasma frequency $\left(\omega_{p}^{\alpha}\right)^{2}$, which can be obtained from first principle

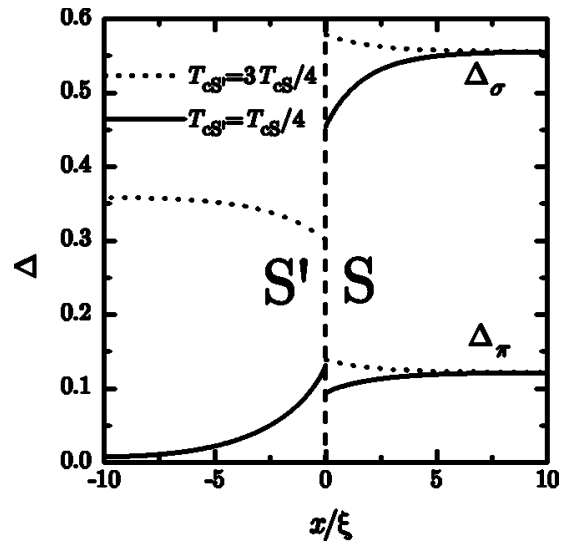

FIG. 1. Pair potential as a function of position for a $S S^{\prime}$ bilayer at $T=0.5 T_{c S}$. The parameters of the bilayer are $\gamma^{\sigma, \pi}=1, \gamma_{B}^{\sigma}=2, \gamma_{B}^{\pi}$ $=1, d_{S} / \xi_{S}=d / \xi=10$, and the coupling constants in the $S$ layer are chosen as expected (Ref. 19) for $\mathrm{MgB}_{2}: \Lambda_{11}=0.81, \Lambda_{22}=0.278$, $\Lambda_{12}=0.115$, and $\Lambda_{21}=0.091$.

calculations. For $\mathrm{MgB}_{2}$, the ratio $R_{B}^{\sigma} / R_{B}^{\pi}=\left(\omega_{p}^{\pi} / \omega_{p}^{\sigma}\right)^{2}$ is 2 and 100 for tunneling in the direction of the $a-b$ plane and $c$ axis, respectively. ${ }^{12}$

In the case of a $S S^{\prime}$ bilayer, the Usadel equation (1) needs to be solved in the $S$ as well as in the $S^{\prime}$ layer, together with the self-consistent determination of the pair potentials in $S$ and $S^{\prime}$, Eq. (2). A general numerical method, using $\Theta$ parametrization, $\Phi=\omega \tan \Theta$, and $G=\cos \Theta$, is described for the single-band case in Ref. 6 . Here, we extended this method by applying the new boundary conditions, Eqs. (3) and (5). The density of states at energy $E$ can be obtained by applying an analytical continuation $\omega=-i E$ to the Usadel equations and the boundary conditions and solving the numerical scheme in the complex energy plane.

The numerically obtained dependence of the pairpotential on position is presented in Fig. 1 for the example in which the coupling constants are taken as calculated for $\mathrm{MgB}_{2}$ in Ref. 19. The parameter values are indicated in the caption. For temperatures above $T_{c S^{\prime}}$ (solid lines in Fig. 1), it can be seen that the pair-potential in $S^{\prime}$ increases towards the interface, while $\Delta_{\sigma}$ decreases, as expected in analogy with the proximity effect in the single-band case. The decrease in $\Delta_{\pi}$ towards the interface can be explained by the relatively strong coupling between the $\sigma$ and $\pi$ bands. By decreasing the interband coupling constants and by increasing the coupling to $S^{\prime}$ (lower interface suppression parameters), one can obtain the opposite regime, in which $\Delta_{\pi}$ increases towards the interface. For relatively large values of $T_{C S^{\prime}}$, and for $T<T_{c S^{\prime}}$, we even predict an increase in $\Delta_{\sigma}$ towards the interface, as illustrated by the dashed line in Fig. 1.

The latter result is quite remarkable since it predicts a phenomenon in which the superconductivity in a two-band superconductor is enhanced by the proximity to a superconductor with a lower transition temperature. The physics of this effect can be explained by considering the presence of $S^{\prime}$ as an additional superconducting band. The coupling between $S^{\prime}$ and the $\pi$ band enhances the superconductivity in the $\pi$ band, while the interband coupling ensures an enhancement of the superconductivity in the $\sigma$ band towards 


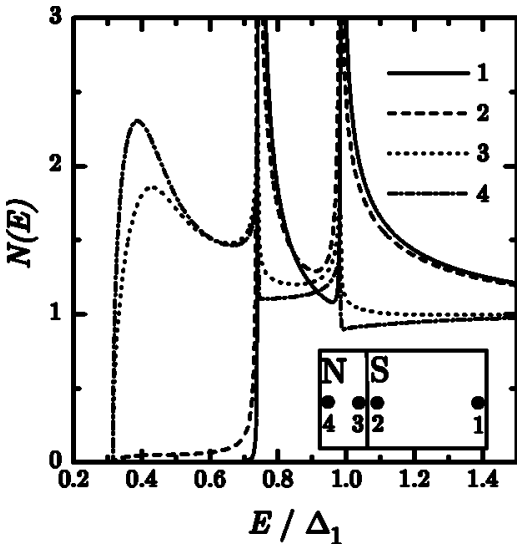

FIG. 2. Normalized density of states in a proximized $S N$ bilayer at several positions in the bilayer (1-4, as indicated in the inset), where $S$ is a two-band superconductor. The parameters of the bilayer are $\gamma^{1,2}=0.1, \gamma_{B}^{1,2}=5, d_{S} / \xi_{S}=10, d / \xi=1$ and the coupling constants in the $S$ layer are chosen as $\Lambda_{11}=0.5, \Lambda_{22}=0.4, \Lambda_{12}=\Lambda_{21}$ $=0.1$.

the $S$ - $S^{\prime}$ interface. From this, it can be seen that the enhancement effect is strongest when the $\sigma$ band is decoupled from the $S^{\prime}$ layer, which is the case for example when the interface normal is parallel to the crystallographic $c$ axis of $\mathrm{MgB}_{2}$, due to the vanishingly small ratio $R_{B}^{\pi} / R_{B}^{\sigma}$ in that case. Note that such an enhancement is a surface effect, while the critical temperature of a $S S^{\prime}$ bilayer is always reduced as compared to $T_{c S}$.

As an example, in Fig. 2 the results of a calculation of the density of states in a $S N$ bilayer are presented. In the considered case, the bulk energy gaps in a two-band superconductor are not too different. As is seen from the figure, the density of states in the $N$ layer has three peaks: the lowest energy peak corresponds to the proximity induced minigap and the two other peaks correspond to the bulk energy gaps in the two-band superconductor $S$. The existence of a minigap is a characteristic feature of the proximity effect in a $S N$-bilayer in the dirty limit, as was studied in detail in the single-band case in Ref. 6. As we can see, the minigap persists in the two-band case as well and its magnitude depends on the parameters of the interface, thicknesses of the $N$ and $S$ and the values of the bulk gaps in the superconductor.

The next step in investigating the influence of multiband superconductivity on the proximity effect is to study supercurrents in multiband proximized structures. We will consider double-barrier structures consisting of two $S$ electrodes coupled by a normal metal $N$. As a model system we use a SINIS double-barrier hybrid structure, since in practical devices interface potential barriers are always present at the $S-N$ interfaces, either originating from a Fermi-velocity mismatch, degradation of surface layers, or artificially deposited oxide barriers.

If the conditions of the dirty limit (electron mean free path $l \ll d, \xi)$ are fulfilled in the $N$ interlayer, than the stationary Josephson effect in the structure can be analyzed in the framework of the Usadel equations by the method developed in Refs. 5 and 17 for the single-band case. We assume that the interface transparencies are small enough such that the condition $1+\gamma_{B 1,2}^{\alpha} \gg \gamma_{1,2}^{\alpha}$ holds at both $N S$ interfaces (here and below we drop the subscript $S$ ). In this case, the suppression of superconductivity in the $S$ layers is weak and the Green's functions in the electrodes near the interfaces $G_{1,2}^{\alpha}$ and $\Phi_{1,2}^{\alpha}$ are equal to their bulk values. To calculate the supercurrent, it is sufficient to consider Eq. (5) at the two interfaces, giving

$$
\xi G \Phi^{\prime}=\sum_{\alpha} \frac{G_{1,2}^{\alpha}}{\gamma_{B 1,2}^{\alpha}}\left( \pm \Phi_{1,2}^{\alpha} \mp \Phi\right), x= \pm \frac{d}{2} .
$$

For simplicity, we will consider symmetric junctions where $G_{1,2}^{\alpha} \equiv G_{S}^{\alpha}$ and $\gamma_{B 1,2}^{\alpha} \equiv \gamma_{B}^{\alpha}$, and where the functions $\Phi_{1,2}^{\alpha}$ are related to the phase shift $\varphi$ across the junction by $\Phi_{1,2}^{\alpha}$ $=\Delta_{\alpha} \exp ( \pm i \varphi / 2)$. Further, we consider purely normal $N$ layer with vanishing pair potential $\Delta=0$ and restrict ourselves to considering the limit of a small interlayer thickness $d \ll \xi$.

In the limit $d \ll \xi$, there are two characteristic frequencies $\Omega_{1,2}$ in the Usadel equations (1) and (2). At $\omega \lesssim \Omega_{1}=\pi T_{c S} \frac{\xi}{d}$ $\gg \Omega_{2}=\pi T_{c S}$ we can neglect all nongradient terms in the Usadel equation. Hence, $\left[G^{2} \Phi^{\prime}\right]^{\prime}=0$, and in the zero approximation on $d / \xi$ one obtaines that all $\Phi$ functions are spatially independent constants $\Phi=A$. In the next approximation we have

$$
\Phi=A+B \frac{x}{\xi}+A \frac{x^{2} \beta^{2}}{2 \xi^{2}}, \beta^{2}=\frac{\omega}{\pi T_{c S} G} .
$$

From the boundary conditions and by taking into account that in our model $\Phi_{1,2}^{\alpha}=\Delta_{\alpha} \exp ( \pm i \varphi / 2)$, we finally will have

$$
\begin{gathered}
A=\frac{\widetilde{\Delta} \eta}{\widetilde{G}}, B=\frac{i \widetilde{\Delta}}{G} \frac{d}{\xi} \sin (\varphi / 2), \\
G=\frac{\omega}{\sqrt{\omega^{2}+A^{2}}}=\frac{\omega \widetilde{G}}{\sqrt{\omega^{2} \widetilde{G}^{2}+\widetilde{\Delta}^{2} \eta^{2}}},
\end{gathered}
$$

where $\gamma_{B M}^{\alpha}=\gamma_{B}^{\alpha} d / \xi, \eta^{2}=\cos ^{2}(\varphi / 2)$ and

$$
\widetilde{G}=\sum_{\alpha} \frac{G_{S}^{\alpha}}{\gamma_{B M}^{\alpha}}+\frac{\omega}{2 \pi T_{c S}}, \tilde{\Delta}=\sum_{\alpha} \frac{G_{S}^{\alpha} \Delta_{\alpha}}{\gamma_{B M}^{\alpha}} .
$$

The density of states $N(E)=\operatorname{Re}(G)$ in the interlayer of the double-barrier junction can now be found from an analytical continuation of Eq. (9) to real energies $\omega=-i E$. The results for the two-band case are plotted in Fig. 3. The known density of states for a single-band SINIS junction ${ }^{21}$ is shown in the inset. For $\gamma_{B M} \ll 1$, the single-band results show a peak in the density of states at $\Delta \cos (\phi / 2)$, while the density of states in the two-band junction in this regime is predicted to have a peak at a value that is even lower than $\Delta_{\pi} \cos (\phi / 2)$. For larger values of $\gamma_{B M}$, the density of states shows three peaks: at the minigap and at $\Delta_{\pi}$ and $\Delta_{\sigma}$, in analogy with the two peaks in the density of states of a single-band SINIS junction.

Substituting Eq. (8) into the supercurrent expression 


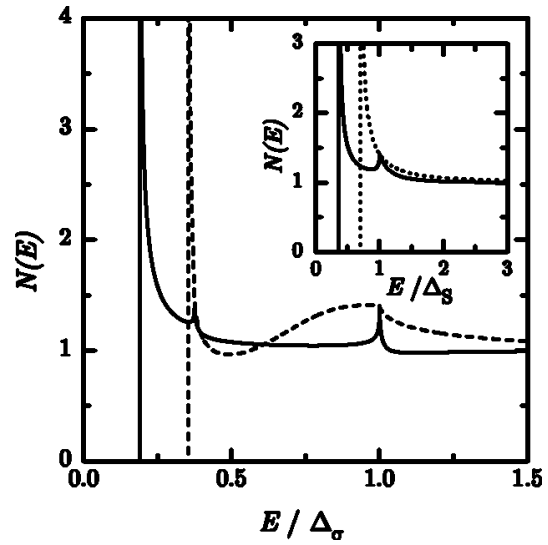

FIG. 3. Normalized density of states in the interlayer of a SINIS double-barrier structure, where $S$ is the two-band superconductor $\mathrm{MgB}_{2}$, and the phase difference over the junction is $\pi / 2$. The density of states is shown for small $\gamma_{B M}$ (dashed line: $\gamma_{B M}^{\sigma}=0.2, \gamma_{B M}^{\pi}$ $=0.1$ ) and large $\gamma_{B M}$ (solid line: $\gamma_{B M}^{\sigma}=20, \gamma_{B M}^{\pi}=10$ ). For comparison, the inset shows the density of states in the interlayer of a SINIS junction with single-band superconductors (solid line: $\gamma_{B M}=2$, dashed line: $\gamma_{B M}=2 \times 10^{-3}$ ).

$$
I=\sigma 2 \pi T \operatorname{Im} \sum_{\omega \geqslant 0} \frac{1}{\omega^{2}} G^{2} \Phi^{*} \Phi^{\prime},
$$

and taking $\Phi^{*} \Phi^{\prime}$ in lowest order equal to $A^{*} B$, we obtain

$$
I=\frac{\pi T}{\xi \rho} \sum_{\omega \geqslant 0} \frac{d \widetilde{\Delta^{2}} \sin (\phi)}{\omega \xi \sqrt{\omega^{2} \widetilde{G}^{2}+\widetilde{\Delta}^{2} \eta^{2}}} .
$$

A generalization to take boundary asymmetry and a finite $\Delta$ in the interlayer into account can be made straightforwardly.

In the two-band case in the limit $\gamma_{B M}^{\sigma} \rightarrow \infty$, which is for example the case for tunneling in the $\mathrm{MgB}_{2} c$-axis direction, the normal metal is only proximized by the $\pi$ gap of electrode $S$ and Eq. (12) gives

$$
I=\frac{\pi T}{\xi \rho \gamma_{B}^{\pi}} \sum_{\omega \geqslant 0} \frac{G_{S}^{\pi} \Delta_{\pi}^{2} \sin (\phi)}{\omega \sqrt{\left[\omega+\frac{\omega^{2} \gamma_{B M}^{\pi}}{2 \pi T_{c S} G_{S}^{\pi}}\right]^{2}+\Delta_{\pi}^{2} \cos ^{2}\left(\frac{\phi}{2}\right)}},
$$

which has been previously obtained ${ }^{5,20}$ for SINIS junctions with single-band superconductivity in $S$.

If there is no superconductivity in one of the bands, Eq. (12) describes the presence of an effective normal shunt connected parallel to the supercurrent. This leads to a reduction of the $I_{c} R_{N}$ product as compared to the case of a SINIS junction with single-band superconductivity in the electrodes.

The temperature dependence of the critical current can now be calculated for SINIS Josephson structures for different orientations of the crystallographical axis with respect to the interface normal. The gap functions $\Delta_{\pi, \sigma}(T)$ and the ratio $\gamma_{B}^{\sigma} / \gamma_{B}^{\pi}$ follow from band structure calculations. ${ }^{12}$ The results

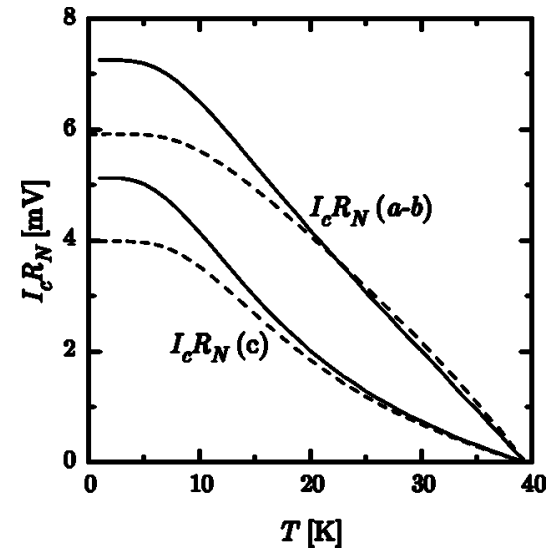

FIG. 4. $I_{C} R_{N}$ for double-barrier $\mathrm{MgB}_{2}$ SINIS junctions in the regime of $\gamma_{B M}^{\sigma, \pi} \ll 1$ (solid lines), compared to $I_{c} R_{N}$ for $\mathrm{MgB}_{2} S I S$ tunnel junctions (Ref. 12) (dashed lines). The total $I_{c} R_{N}$ of $a-b$ plane $\operatorname{Mg} B_{2}$ junctions is an average over all bands, while $c$-axis junctions only contain a $\pi$-band contribution.

are shown in Fig. 4 for vanishingly small $\gamma_{B M}$, and compared to the calculation results for $S I S$ junctions. ${ }^{12}$ The full specific interface resistance of a SINIS junction $R_{N}=R_{B}^{\sigma} R_{B}^{\pi} /\left[R_{B}^{\sigma}\right.$ $\left.+R_{B}^{\pi}\right]$. It is clearly seen that the critical current of SINIS junctions is larger than in SIS structures, practically in the whole temperature region, as is the case for single band superconductors. ${ }^{5,20}$ At low temperatures the $I_{c} R_{N}$ product can be as large as $5.2 \mathrm{mV}$ when only the $\pi$ band contributes to the current and close to $7.3 \mathrm{mV}$ when the sum over different band contributions can be taken into account, as is the case for tunneling in the direction of the $a-b$ plane. The negative curvature of $I_{c} R_{N}(T)$ is a direct consequence of the two-band nature of superconductivity and is absent in $I_{C} R_{N}(T)$ of single-band SINIS junctions in the regime of small $\gamma_{B M}{ }^{20}$

In summary, we have formulated a microscopic theory of the proximity effect in hybrid structures based on multiband superconductors in the diffusive limit. We have shown that the existence of multiple superconducting bands manifests itself in the proximity effect between a normal metal and a superconductor as the occurence of additional peaks in the density of states at the normal metal side. The interplay between the proximity effect and interband coupling determines the gap magnitudes at the interfaces. We predict an enhancement of superconductivity at the surface of a multiband superconductor by the proximity to a superconductor with a lower transition temperature. The supercurrent in multiband SINIS Josephson junctions was calculated and compared to known single-band results and predictions for multiband tunnel junctions.

We acknowledge useful discussions with O.V. Dolgov and A.E. Koshelev and support from INTAS Projects Nos. 2001-0617 and 2001-0809. M.Yu.K. acknowledges support from the Russian Ministry of Industry, Science and Technology. 
${ }^{1}$ L. N. Cooper, Phys. Rev. Lett. 6, 689 (1961).

${ }^{2}$ N. R. Werthamer, Phys. Rev. 132, 2440 (1963).

${ }^{3}$ P. G. de Gennes, Rev. Mod. Phys. 36, 225 (1964).

${ }^{4}$ W. L. McMillan, Phys. Rev. 175, 537 (1968).

${ }^{5}$ M. Yu. Kupriyanov and V. F. Lukichev, Zh. Eksp. Teor. Fiz. 94, 139 (1988) [Sov. Phys. JETP 67, 1163 (1988)].

${ }^{6}$ A. A. Golubov, E. P. Houwman, J. G. Gijsbertsen, V. M. Krasnov, J. Flokstra, H. Rogalla, and M. Yu. Kupriyanov, Phys. Rev. B 51, 1073 (1995).

${ }^{7}$ Ya. V. Fominov and M. V. Feigel'man, Phys. Rev. B 63, 094518 (2001).

${ }^{8}$ C. W. J. Beenakker, Rev. Mod. Phys. 69, 731 (1997).

${ }^{9}$ W. Belzig, F. K. Wilhelm, C. Bruder, G. Schön, and A. D. Zaikin, Superlattices Microstruct. 25, 1251 (1999).

${ }^{10}$ Physica C 385 (1-2) (2003) (Special issue on $\mathrm{MgB}_{2}$ ).

${ }^{11}$ A. Y. Liu, I. I. Mazin, and J. Kortus, Phys. Rev. Lett. 87, 087005 (2001).

${ }^{12}$ A. Brinkman, A. A. Golubov, H. Rogalla, O. V. Dolgov, J. Kortus, Y. Kong, O. Jepsen, and O. K. Andersen, Phys. Rev. B 65,
180517 (2002).

${ }^{13}$ J.-I. Kye, H. N. Lee, J. D. Park, S. H. Moon, and B. Oh, IEEE Trans. Appl. Supercond. 13, 1075 (2003).

${ }^{14}$ K. D. Usadel, Phys. Rev. Lett. 25, 507 (1970).

${ }^{15}$ I. I. Mazin, O. K. Andersen, O. Jepsen, O. V. Dolgov, J. Kortus, A. A. Golubov, A. B. Kuz'menko, and D. van der Marel, Phys. Rev. Lett. 89, 107002 (2002).

${ }^{16}$ A. E. Koshelev and A. A. Golubov, Phys. Rev. Lett. 90, 177002 (2003).

${ }^{17}$ A. V. Zaitsev, Zh. Eksp. Teor. Fiz. 86, 1742 (1984) [Sov. Phys. JETP 59, 1015 (1984)].

${ }^{18}$ I. I. Mazin, Phys. Rev. Lett. 83, 1427 (1999).

${ }^{19}$ A. A. Golubov, J. Kortus, O. V. Dolgov, O. Jepsen, Y. Kong, O. K. Andersen, B. J. Gibson, K. Ahn, and R. K. Kremer, J. Phys.: Condens. Matter 14, 1353 (2002).

${ }^{20}$ M. Yu. Kupriyanov, A. Brinkman, A. A. Golubov, M. Siegel, and H. Rogalla, Physica C 326-327, 16 (1999).

${ }^{21}$ A. Brinkman, A. A. Golubov, H. Rogalla, F. K. Wilhelm, and M. Yu. Kupriyanov, Phys. Rev. B 68, 224513 (2003). 